\title{
Stress is irrelevant to the onset and exacerbation of sensorineural hearing loss: Evaluation using various types of stress models in mice
}

\author{
Yamaguchi T*, Ogita K, Yoneyama M and Onaka Y \\ Laboratory of Pharmacology, Faculty of Pharmaceutical Sciences, Setsunan University, Hirakata, Osaka, Japan
}

\begin{abstract}
Chronic stress has been argued to produce sensorineural hearing loss in human-beings. To elucidate the influence of stress on hearing loss, we tested changes in hearing ability in various types of stress-model mice, such as water-immersion restraint stress (WIRS, $3 \mathrm{~h} /$ day, for 4 weeks), social defeat stress (SDS, $10 \mathrm{~min} /$ day, for 2 weeks), and social isolation stress (SIS, for 4 weeks). No marked changes in hearing ability and the number of cochlear hair cells lost were observed in mice exposed to these different types of stress.In addition, these stresses did not exacerbate temporary hearing impairment induced by noise $(8 \mathrm{kHz}$ octave band noise, $90 \mathrm{~dB}$ sound pressure level, $1 \mathrm{~h}$ ). Taken together, our data suggest that chronic stress did not cause hearing loss in mice. These findings reveal that chronic stress is irrelevant to the onset and exacerbation of sensorineural hearing loss at least in mice.
\end{abstract}

\section{Introduction}

Human beings are simultaneously exposed to social stress in their daily lives. Repeated exposure to social and psychological stress has been shown to disturb health, and is considered to cause lifestyle-related diseases, such as hyperinsulinemia, hyperglycemia, cardiovascular diseases, and obesity [1,2]. Additionally, the involvement of stress in the development of psychiatric disorders, such as depression, has been widely investigated in animals $[3,4]$. Moreover, several studies have proposed that exposure to chronic social stress produces sudden sensorineural hearing loss (SNHL) in human beings [5,6].

Sudden SNHL is an acute dysfunction of the inner ear that is characterized by sudden hearing loss and potential progression to complete deafness. Elderly people are primarily affected, but it can occur at any age. Sudden SNHL is usually classified as idiopathic, because the causative factor is not identified in most cases [7-10]. Impaired hearing ability greatly detracts from the quality of life of individuals, and may trigger psychiatric disorders, such as cognitive dysfunction [11,12]. Moreover, since most SNHL induced byinner eardysfunctionisirreversible, SNHL is becoming aglobal social problem.

The present study aimed to test the hypothesis that chronic stress increases the risk of developing hearing loss. In addition, we set out to verify whether noise-exposure hearing loss is exacerbated by chronic stress.

\section{Materials and methods}

Animals: All experiments used here met the Guidelines of the Japanese Society for Pharmacology and were approved by the Committee for Ethical Use of Experimental Animals at Setsunan University. Adult male Std-ddY mice (SHIMIZU Laboratories Supplies Co., Ltd, Kyoto, Japan), weighing 22-26 g, were used for preparation of stress models.
Stress treatment: Figure 1 shows schedules for stress treatment; water-immersion restraint stress (WIRS), social defeat stress (SDS), and social isolation stress (SIS). Mice were individually housed for at least 1 week to habituate before commencing experiments. To produce WIRS over a period of 4 weeks, mice were caged in $50 \mathrm{ml}$ polypropylene tubes to restrict their activity and were then immersed vertically for $3 \mathrm{~h}$ every day in $25 \pm 1^{\circ} \mathrm{C}$ water bath cages, in which the water level was kept at shoulder level for the mice. For SDS, mice were individually introduced to the home cage of an unfamiliar aggressive resident mouse for $10 \mathrm{~min}$, and were then kept in a small plastic cage that enabled visual, olfactory, and auditory contact in the home cage of the resident mouse for the remaining time of the day. These mice were

Correspondence to: Yamaguchi T, Laboratory of Pharmacology, Faculty of Pharmaceutical Sciences, Setsunan University45-1 Nagaotoge-cho, Hirakata, Osaka 573-0101, Japan,Tel: 81-(0)72-866-3109; E-mail: t-yamagu@pharm.setsunan.ac.jp

Key words: chronic stress, noise exposure, sensorineural hearing loss

Special Issue: Academic seeds for drugs

Katsura Takano

Associate Professor

Division of Veterinary Science

Graduate School of Life and Environmental Sciences

Osaka Prefecture University

Japan

Yasu-Taka Azuma, Ph.C., Ph.D.

Associate Professor

Principal Investigator at Laboratory on Veterinary Pharmacology Osaka Prefecture University

Japan

Received: June 14, 2017; Accepted: July 06, 2017; Published: July 10, 2017 
(a) WIRS

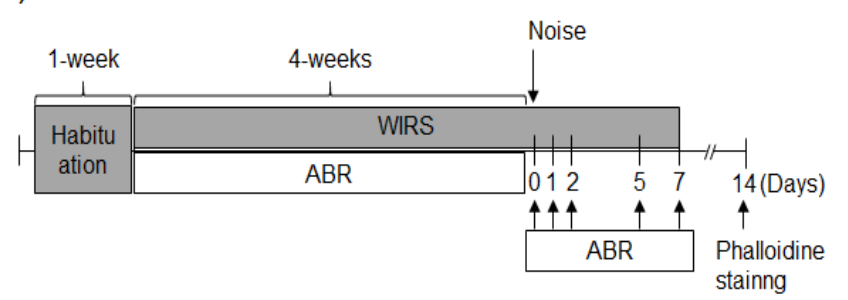

(b) SDS

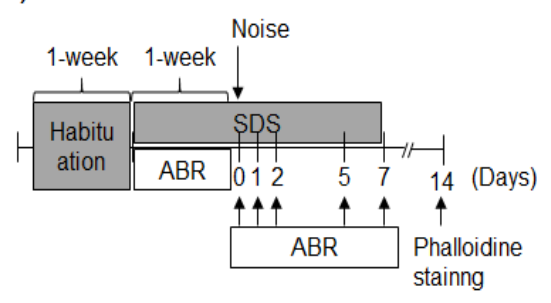

(c) SIS

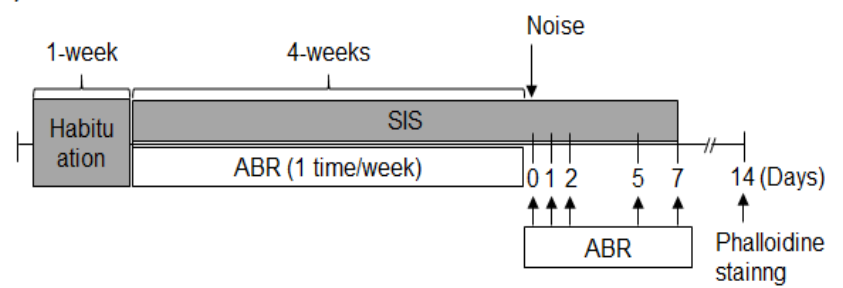

Figure 1. Experimental schedules.All animals underwent 1 week of habituation before stress treatment. (a) Water-immersion restraint stress (WIRS): animals treated with WIRS for 4 weeks were subjected to measurement of auditory brainstem response (ABR) and body weight. (b) Social defeat stress (SDS): animals were exposed to SDS for 1 week and then subjected to measurement of ABR and body weight. (c) Social isolation stress (SIS): animals were treated with SIS for 4 weeks and then subjected to measuring of ABR and body weight.

subjected to social defeat for 7 consecutive days.Control mice were kept alone in transparent plastic cages for 7 consecutive days. For SIS, mice were housed individually for 4 weeks.

Auditory brainstem response (ABR) recording: To determine the hearing ability of the animals, we measured their ABR under anesthesia induced by chloral hydrate $(500 \mathrm{mg} / \mathrm{kg}$, i.p.), as previously reported [13]. The threshold of ABR was determined at the frequencies of 4, 12, and $20 \mathrm{kHz}$, by means of a 5-dB SPL minimum-size step-down from the maximum amplitude. The hearing threshold was defined as the lowest stimulus intensity that produced wave I in the ABR test.

Noise-induced hearing impairment: To establish noise-induced hearing impairment, the sound was generated and amplified as previously described [13]. Each animal was placed in a cage. The mice were then exposed to an octave-band noise, centered at $8 \mathrm{kHz}$, at a 90 $\mathrm{dB}$ sound pressure level, for $1 \mathrm{~h}$, within a sound chamber. As a control, naïve animals were placed in the same cage, but without the noise.

Quantitative assessment of hair cell loss: After the final ABR test, animals were decapitated and cochleae were immersion-fixed with $4 \%$ paraformaldehyde in $0.1 \mathrm{M}$ phosphate buffer ( $\mathrm{pH}$ 7.4) for $2 \mathrm{~h}$ at room temperature after rapid removal of the temporal bone and opening of the round and oval windows. The cochleae were then decalcified in 4\% EDTA solution at room temperature for at least 2-7 days. Under a microscope, the specimens including organ of Corti were dissected in phosphate-buffered saline foe whole-mount cochlear processing. To visualize hair cells, we incubated the specimens with a solution containing $0.3 \%$ Triton $\mathrm{X}-100$ and Alexa-Fluor 568-conjugated phalloidin (1:100 dilution; Invitrogen, Carlsbad, CA, USA) for 30 min at room temperature. The stained specimens were observed under a confocal fluorescence microscope using the FV1000D system (Olympus, Tokyo, Japan) for counting the numbers of missing hair cells in the cochleae. The ratio of missing-to-total hair cells was expressed as a percentage.

Data analysis: The results are presented as the means \pm S.E.M from several separate experiments. Differences were assessed by using analysis of variance. Data were analyzed by means of one-way ANOVA, and a least significant difference post-hoc test was used to evaluate the statistical differences between groups.

\section{Results}

Changes in body weight after stress treatment: To confirm whether animals were exposed to sufficient stress under the current experiments, we measured the body weight of animals during the stress treatments. In mice treated with WIRS or SDS, the body weight decreased markedly during the treatment.However, SIS-exposed mice demonstrated a significant increase in body weight during the treatment (Figure 2).

(a) WIRS

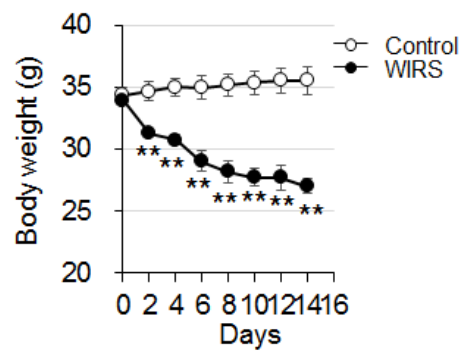

(b) SDS

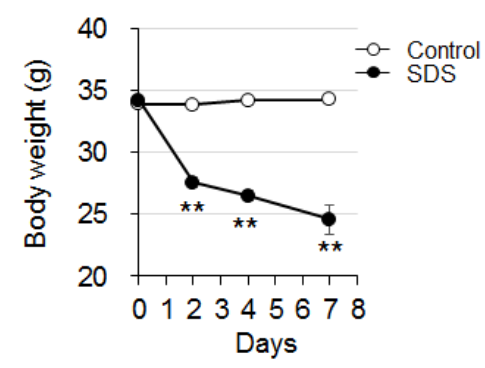

(c) SIS

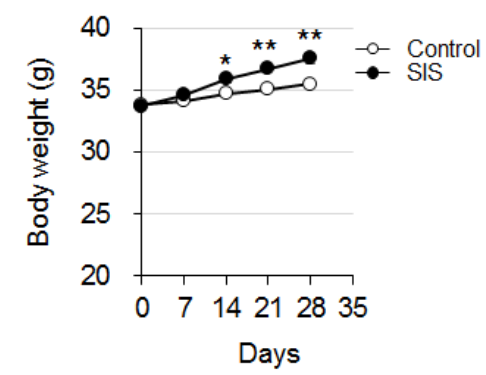

Figure 2. Effect of stress treatment on body weight.Graphs denote the average of body weight.Values are the means \pm S.E.M. from 4 independent experiments performed under the same experimental conditions. ${ }^{* *} \mathrm{P}<0.01$, significantly different from the value obtained for animals not exposed to stress treatment. 
Effect of stress treatment on hearing ability: To assess if physical or social stress increases the risk of developing hearing loss, hearing ability was determined in mice treated with WIRS, SDS, and SIS (Figure 3). No significant impairment in hearing ability occurred at 4, 12, and 20 $\mathrm{kHz}$ in any of the stress groups (WIRS, SDS, and SIS).

Effect of stress treatment on noise-induced temporary hearing impairment: We next assessed the effect of stress treatment on noiseinduced temporary hearing impairment (Figure 4). The ABR threshold was assessed at the frequencies of 4,12 , and $20 \mathrm{kHz}$ on immediately after (day 0) or 7 days after noise exposure. Exposure to noise at a 90$\mathrm{dB}$ sound pressure level for $1 \mathrm{~h}$ produced a temporary threshold shift in ABR immediately after noise exposure. Already on day 1 after noise exposure, ABR returned to the level of animals without exposure to noise.WIRS, SDS, or SIS did not exacerbate the noise-induced hearing impairment, at least on the days tested.

Effect of stress treatment on cochlear outer hair cells damaged after noise exposure: To examine whether stress treatment exacerbates hair cell damage induced by noise exposure, we stained and counted the damaged outer hair cells in the cochlea of noise-exposed animals (Figure 5). In animals exposed to noise alone, $2-4 \%$ of outer hair cells in the cochlea disappeared. The damage to the outer hair cells was not significantly exacerbated by WIRS, SDS, or SIS.

\section{(a) WIRS}

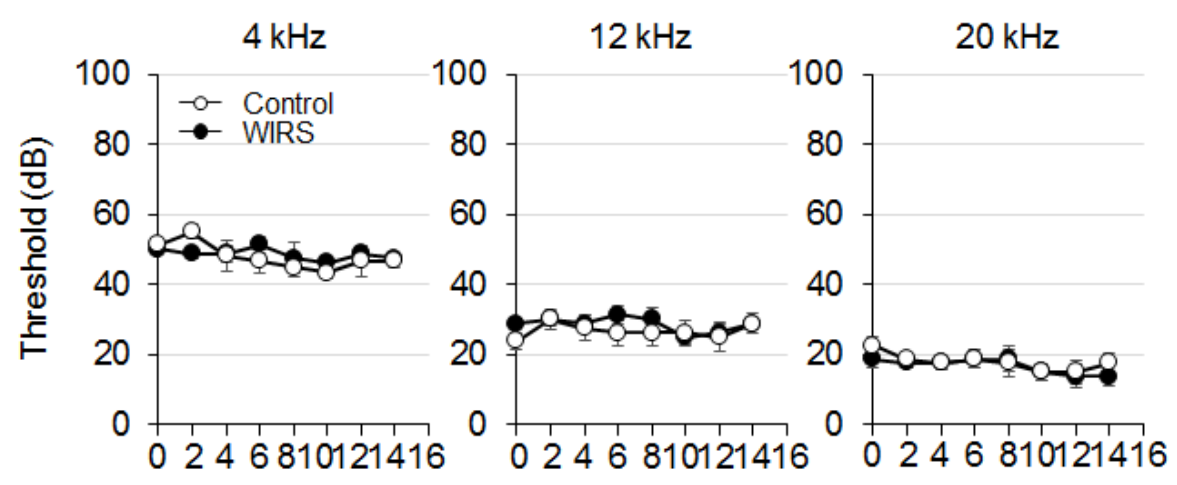

\section{(b) SDS}

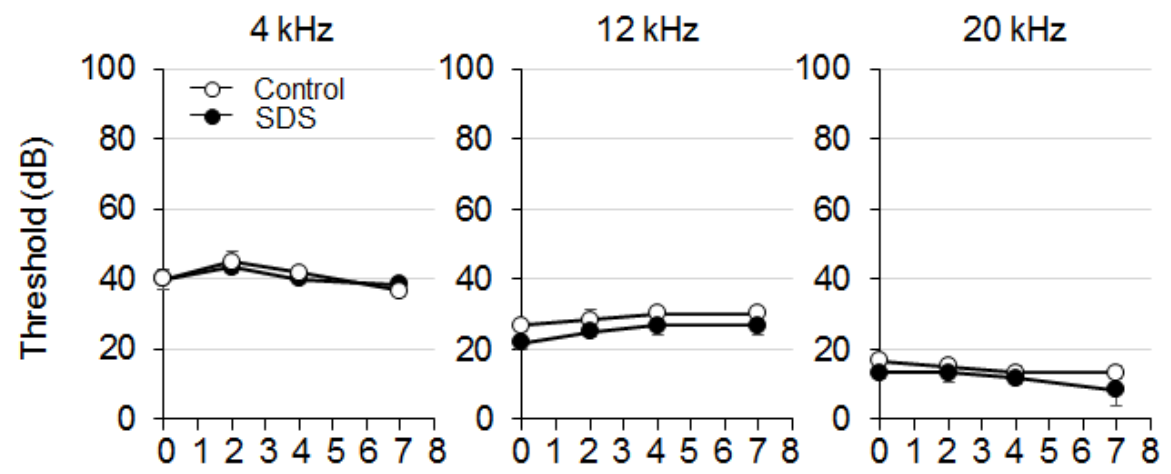

\section{(c) SIS}

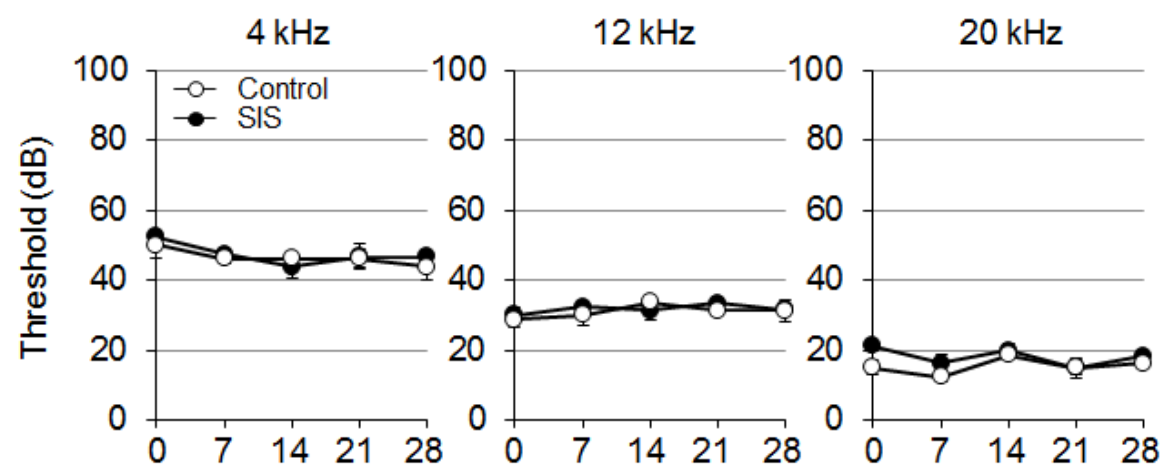

Figure 3. Effect of stress treatment on hearing ability.Animals were treated with stress and then the auditory brainstem response (ABR) threshold was measured.Graphs denote the average of threshold shift at each frequency.Values are the means \pm S.E.M. from 4 independent experiments performed under the same experimental conditions. 
(a) WIRS

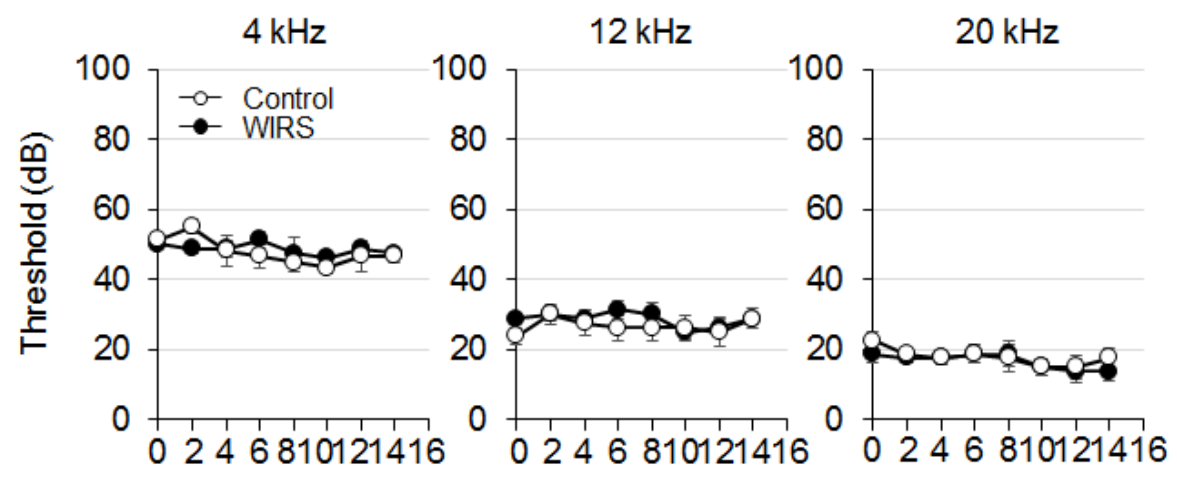

(b) SDS

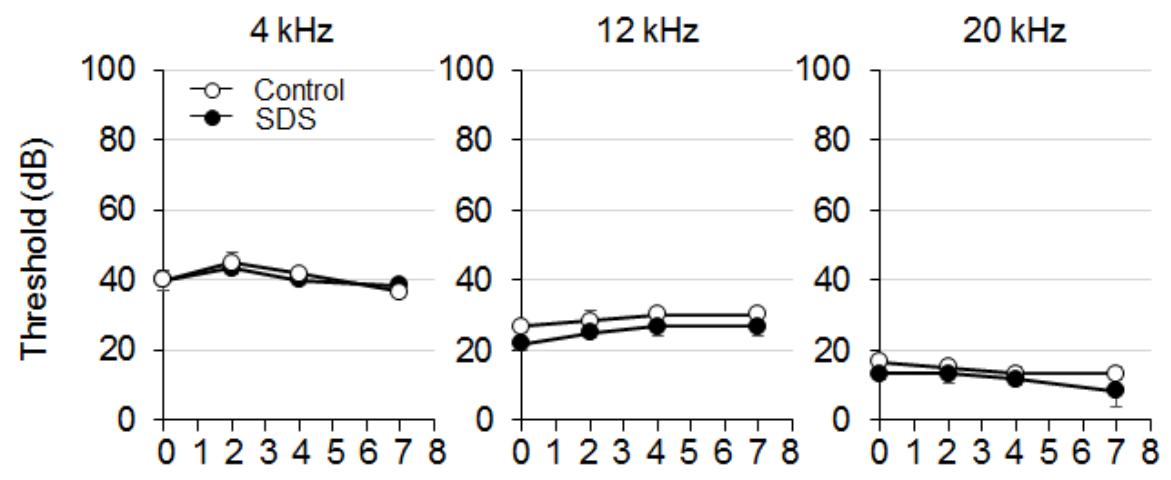

(c) SIS

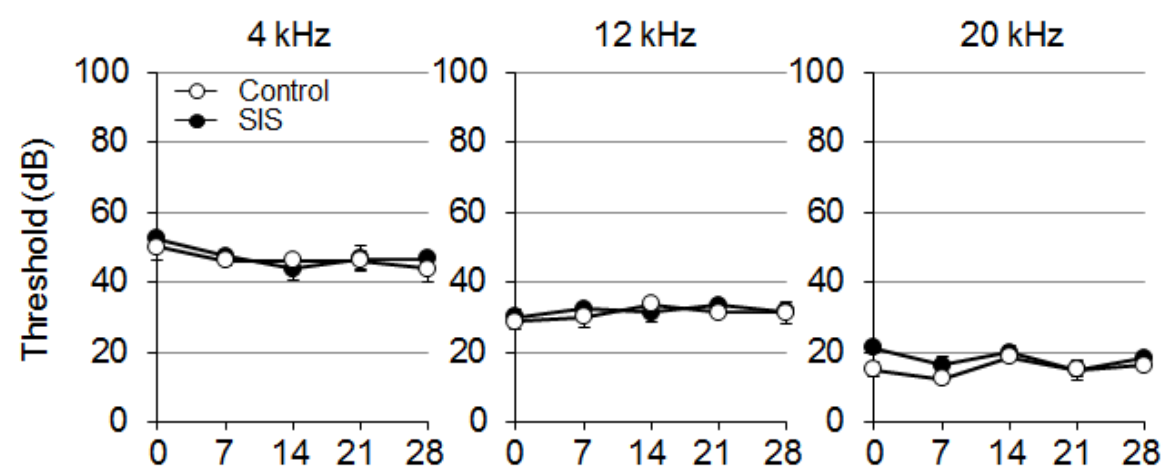

Figure 4. Effect of stress treatment on noise-induced hearing impairment.Animals were exposed to noise at 90-dB SPL for $1 \mathrm{~h}$, after which the auditory brainstem response (ABR) threshold was measured.Graphs denote the average of the threshold shift at each frequency. Values are the means \pm S.E.M. from 4 independent experiments performed under the same experimental conditions.

\section{Discussion}

In this study, we evaluated whether physical and social stresses are involved in the onset or exacerbation of SNHL. Using various types of stress models in mice, our current study demonstrated that exposure to severe stress was not accompanied by impairment of hearing ability. In addition, evidence for the irrelevance of severe stress to the exacerbation of noise-induced hearing loss came from current findings that severe stress treatment produced no significant effect on the temporary threshold shift of ABR and the hair cell loss in mice exposed to noise at a $90-\mathrm{dB}$ sound pressure level. Therefore, the present study, using stress model mice, could not demonstrate data supporting the previous hypothesis that chronic stress increases the risk of developing hearing loss.

We first performed experiments to confirm whether animals were exposed to sufficient stress during our experimental procedures. Changes in the body weight of animals have been reported to be a convenient index for stress exposure [14,15]. A marked decrease in the body weight occurs in WIRS- and SDS-exposed animals [16]; in contrast, SIS promotes food intake and body weight gain [15,17]. These previous reports strongly support that in the present study, animals exposed to WIRS, SDS, or SIS were useful as stress models.

In these stress model animals, hearing impairment and cochlear 
(a)
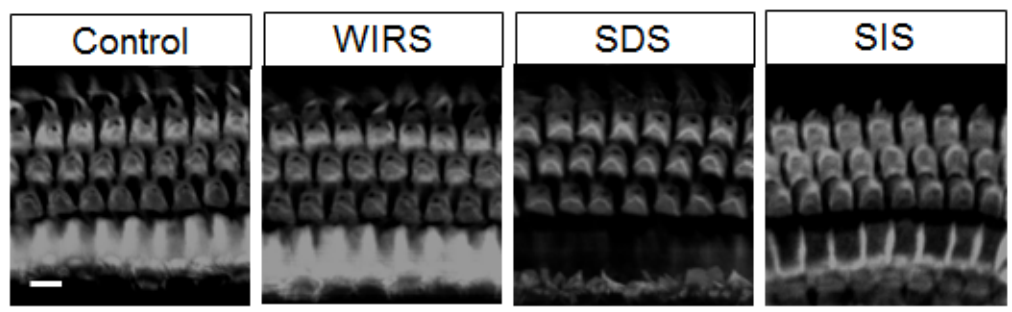

(b)
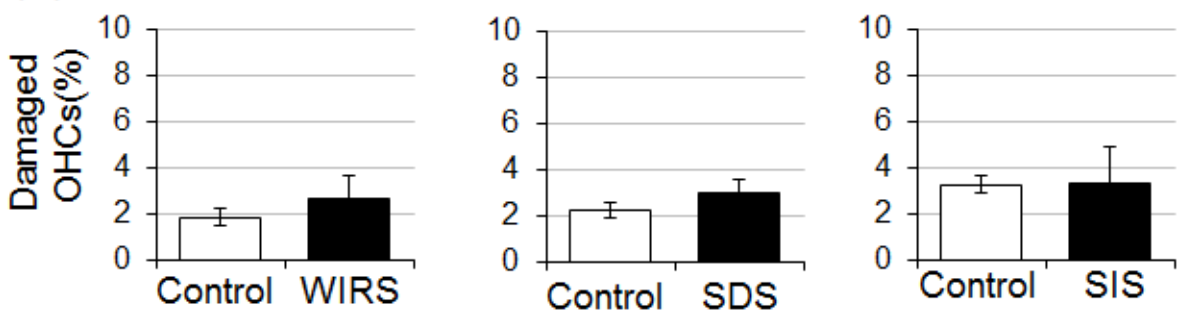

Figure 5. Effect of stress treatment on noise-induced cochlear hair cell loss.Animals were exposed to noise at 90-dB SPL for 1 h.On 7 days after noise exposure, animals were sacrificed, and the inner ear fixed for dissection of the organ of Corti, which was subsequently stained with Alexa-Fluor 568-conjugated phalloidin.(a) Typical fluorescent images of outer hair cells (OHCs) of the middle turn in the organ of Corti.(b) Graphs of the percentage of damaged outer hair cells per the total number of hair cells in all turns of the organ of Corti.Values are the means \pm S.E.M. from 4 independent experiments performed under the same experimental conditions. Scale bar $=10 \mu \mathrm{m}$.

hair cell damage was not observed, at least during the periods tested. There are some possible reasons; the experimental term may have been too short to affect hearing function, although stress-induced physical responses were sufficient as stress models; furthermore, a single stress did not present a necessary and sufficient condition to affect hearing. In human beings, stress exposure in modern society is often of mixed types. Hence, hearing loss might be caused by exposure to a combination of stresses over a long period of time.

Most hearing impairment in adults is SNHL, most of which is induced by inner ear dysfunction, which is irreversible. Cochlear hair cells and nerves, which convert mechanical vibrations into electrical signals that are transmitted to the central nervous system, have extremely poor regenerative ability.It thus is impossible to regenerate them when cell death occurs in the mammalian ear [18]. In noiseexposed humans and animals, acoustic trauma (noise-induced hearing loss) leads to hair cell damage and causes a threshold shift $[19,20]$. Recent studies have indicated that ageing and/or moderate noise exposure can result in hidden hearing loss [21-23], which causes difficulties in speech discrimination and temporal processing in a noisy environment [24]. It can be detected physiologically, and is characterized by reduced amplitude in the sound-evoked spiral ganglion neuron activity (the first peak of the ABR waveform) [25-27]. Therefore, stress-exposed mice in the present study may have characteristics of hidden hearing loss, but further studies would be needed to elucidate such a hypothesis.

In conclusion, the present study clearly demonstrated that chronic stress had no effect on the hearing ability of mice. These results suggest that stress is unlikely to be involved as a cause of SNHL. However, further studies are needed to assess whether stress increases the risk of developing hearing loss in human beings.

\section{Acknowledgements}

This work was supported in part by grants-in-aid for Challenging
Exploratory Research to K.O. (No. 25670042) from the Japan Society for the Promotion of Science.

\section{Conflicts of interest}

The authors declare that they have no conflicts of interest in this research.

\section{References}

1. Rosengren A, HawkenS, Ounpuu S, Sliwa K, Zubaid M, et al. (2004) Association of psychosocial risk factors with risk of acute myocardial infarction in 11119 cases and 13648 controls from 52 countries (the INTERHEART study): case-control study. The Lancet (London, England) 364: 953-962. [Crossref]

2. Orth-Gomer K, Wamala SP, Horsten M, Schenck-Gustafsson K, Schneiderman N, et al. (2000) Maritalstress worsens prognosis in women with coronary heart disease: The Stockholm Female Coronary Risk Study. Jama 284: 3008-3014. [Crossref]

3. Onaka Y, Shintani N, Nakazawa T, Haba R, Ago Y, et al. (2015) CRTH2, a prostaglandin D2 receptor, mediates depression-related behavior in mice. Behav Brain Res 284: 131137. [Crossref]

4. Liu YY, Zhou XY, Yang LN, Wang HY, Zhang YQ, et al. (2017) Social defeat stress causes depression-like behavior with metabolite changes in the prefrontal cortex of rats. PloS one 12: e0176725. [Crossref]

5. Joo YH, Han KD, Park KH (2015) Association of Hearing Loss and Tinnitus with Health-Related Quality of Life: The Korea National Health and Nutrition Examination Survey. PloS one 10: e0131247. [Crossref]

6. Malina CR (2015) Chronic occupational stress exposure may increase the vulnerability to acoustic trauma in military professionals. Revista medico-chirurgicala a Societatii de Medici siNaturalisti din Iasi 119: 51-54. [Crossref]

7. Koc A, Sanisoglu O (2003) Sudden sensorineural hearing loss: literature survey on recent studies. The Journal of otolaryngology 32: 308-313. [Crossref]

8. Rudack C, Langer C, Stoll W, Rust S, Walter M (2006) Vascular risk factors in sudden hearing loss. ThrombHaemost 95: 454-461. [Crossref]

9. Chau JK, Lin JR, Atashband S, Irvine RA, Westerberg BD (2010) Systematic review of the evidence for the etiology of adult sudden sensorineural hearing loss. The Laryngoscope 120: 1011-1021. [Crossref] 
10. Masuda M, Kanzaki S, Minami S, Kikuchi J, Kanzaki J, et al. (2012) Correlations of inflammatory biomarkers with the onset and prognosis of idiopathic sudden sensorineural hearing loss. Otol Neurotol 33: 1142-1150. [Crossref]

11. Mitoku K, Masaki N, Ogata Y, Okamoto K (2016) Vision and hearing impairments, cognitive impairment and mortality among long-term care recipients: a populationbased cohort study. BMC Geriatrics 16: 112. [Crossref]

12. Pichora-Fuller MK (2015) Cognitive decline and hearing health care for older adults. AmJ Audiol 24: 108-111. [Crossref]

13. Nagashima R, Yamaguchi T, Tanaka H, Ogita K (2010) Mechanism underlying the protective effect of tempol and Nomega-nitro-L-arginine methyl ester on acoustic injury: possible involvement of c-Jun N-terminal kinase pathway and connexin26 in the cochlear spiral ligament. J Pharmacol Sci 114: 50-62. [Crossref]

14. Yamada C, Saegusa Y, Nahata M, Sadakane C, Hattori T, et al. (2015) Influence of Aging and Gender Differences on Feeding Behavior and Ghrelin-Related Factors during Social Isolation in Mice. PloS one 10: e0140094. [Crossref]

15. Hermes G, Li N, Duman C, Duman R (2011) Post-weaning chronic social isolation produces profound behavioral dysregulation with decreases in prefrontal cortex synaptic-associated protein expression in female rats. Physiol Behav 104: 354-359. [Crossref]

16. Pulliam JV, Dawaghreh AM, Alema-Mensah E, Plotsky PM (2010) Social defeat stress produces prolonged alterations in acoustic startle and body weight gain in male Long Evans rats. J Psychiatr Res 44: 106-111. [Crossref]

17. Sakakibara H, Suzuki A, Kobayashi A, Motoyama K, Matsui A, et al. (2012) Social isolation stress induces hepatic hypertrophy in C57BL/6J mice. J Toxico Sci 37: 10711076. [Crossref]
18. Fujioka M, Okano H, Edge AS (2015) Manipulating cell fate in the cochlea: a feasible therapy for hearing loss. Trends neurosci 38: 139-144. [Crossref]

19. Schmiedt RA (1984) Acoustic injury and the physiology of hearing. J Acoust Soc Am 76: 1293-1317. [Crossref]

20. Yamaguchi T, Yoneyama M, Ogita K (2017) Calpain inhibitor alleviates permanen hearing loss induced by intense noise by preventing disruption of gap junctionmediated intercellular communication in the cochlear spiral ligament. Eur J Pharmacol 803: 187-194. [Crossref]

21. Suzuki J, Corfas G, Liberman MC (2016) Round-window delivery of neurotrophin 3 regenerates cochlear synapses after acoustic overexposure. SciRep 6: 24907. [Crossref]

22. Liberman MC, Epstein MJ, Cleveland SS, Wang H, Maison SF (2016) Toward a Differential Diagnosis of Hidden Hearing Loss in Humans. PloS one 11: e0162726. [Crossref]

23. Viana LM, O'Malley JT, Burgess BJ, Jones DD, Oliveira CA, et al. (2015) Cochlear neuropathy in human presbycusis: Confocal analysis of hidden hearing loss in postmortem tissue. HearRes 327: 78-88. [Crossref]

24. Liberman MC (2015) Hidden hearing loss. Scientific American 313: 48-53. [Crossref]

25. Bharadwaj HM, Verhulst S, Shaheen L, Liberman MC, Shinn-Cunningham BG (2014) Cochlear neuropathy and the coding of supra-threshold sound. Front Syst Neurosci 8 : 26. [Crossref]

26. Mehraei G, Hickox AE, Bharadwaj HM, Goldberg H, Verhulst S, et al. (2016) Auditory Brainstem Response Latency in Noise as a Marker of Cochlear Synaptopathy. $J$ Neurosci 36: 3755-3764. [Crossref]

27. Sergeyenko Y, Lall K, Liberman MC, Kujawa SG (2013) Age-related cochlear synaptopathy: an early-onset contributor to auditory functional decline. J Neurosci 33 : 13686-13694. [Crossref]

Copyright: (C2017 Yamaguchi T. This is an open-access article distributed under the terms of the Creative Commons Attribution License, which permits unrestricted use, distribution, and reproduction in any medium, provided the original author and source are credited. 